\title{
Weight Calculation And Purity Identification Of Symplectic Self-orthogonal Codes
}

\author{
Cao Kai, Guo Luobin, Li Chaoyang, Zheng Yuxiang, He Leinan, Zhao Zhenpeng \\ College of Science, Air Force Engineering University \\ Xi'an, shaanxi, P.R. China \\ glbglb2011@163.com
}

\begin{abstract}
The purity identification of quantum errorcorrecting codes and symplectic self-orthogonal is a pivotal problem in quantum code theory. This work is dedicated to some issues of symplectic codes. First, by the MacWillams identity, a fast algorithm to determine the weight polynomial of high-dimension symplectic codes is designed; second a solution to seek for the dual codes of symplectic codes is derived; third the effective method to identify the purity of symplectic codes is obtained; and at last constructions of symplectic selforthogonal codes are given, resulted three impure symplectic self-orthogonal codes, the quantum codes stabilized by the three impure symplectic self-orthogonal codes are optimal. The above work may offer new methods for purity identification of symplectic self-orthogonal codes and quantum error-correcting codes.
\end{abstract}

Keywords-quantum error-correcting codes; symplectic selforthogonal code; weight polynomial; pure codes

\section{INTRODUCTION}

Symplectic codes present itself with the stabilizer quantum error-correcting codes(QECCs)[1]. In 1998, Calderbank, Rains, Shor and Sloane established the connection between symplectic codes and QECCs, and they proved that each symplectic self-orthogonal code corresponds to a stabilizer quantum error-correcting code uniquely [2]. Laterly, the class of symplectic codes that can be converted to linear codes have been determined based on some basic principles in linear coding theory. Research on the distance and weight distribution properties of this special class of symplectic self-orthogonal codes contributed to quantum code constructions. While as time goes by, there still lacks of a promising generic theory and method in weight calculation and purity identification of symplectic self-orthogonal codes, restricted the development of quantum coding theory. Then this paper is dedicated to such related problems.

\section{BASIC CONCEPTS ON SYMPLECTIC CODES}

Let $F_{2}=\{0,1\}$ be the binary field with two elements, $\mathrm{F}_{2}^{2 \mathrm{n}}$ the $2 n$-dimensional space over $F_{2}$. Each vector in $\mathrm{F}_{2}^{2 \mathrm{n}}$ has the form $\alpha=(a \mid b)$, where $a, b$ belong to $\mathrm{F}_{2}^{\mathrm{n}}$, the symplectic weight of $\alpha=(a \mid b)$ is defined as

$$
w t s(\alpha)=w t s(a \mid b)=\left\{i \mid\left(a_{i}, b_{i}\right) \neq(0,0)\right\} \mid
$$

Suppose $\alpha=(a \mid b), \beta=\left(a^{\prime} \mid b^{\prime}\right)$ in $F_{2}^{n}$, then symplectic inner product of $\alpha$ and $\beta$ is defined as

$$
(\alpha, \beta)_{s}=a b^{\prime T}+a^{\prime} b^{T}
$$

When $(\alpha, \beta)_{s}=0$, then $\alpha$ and $\beta$ are called orthogonal vectors.

An $m$-dimensional subspace $C$ of $\mathrm{F}_{2}^{2 \mathrm{n}}$ is called an $m$ dimensional symplectic code, it can be denoted as $C=[2 n, m]_{s}$, and vector in $C$ are called codewords.

If there are $A_{i}$ codewords of symplectic weight $i$, then the polynomial $W_{s}(z)=\sum_{i=0}^{n} A_{i} z^{i}$ is the symplectic-weight polynomial of the symplectic code . The dual code of $C$ is $C^{\perp s}=\left\{\alpha \mid(\alpha, \beta)_{s}=0, \forall \beta \in C\right\}$. And when $C \subset C^{\perp s}$ then $C$ is a symplectic self-orthogonal code.

Symplectic self-orthogonal code is a base in constructing QECCs, and is a main topic in considerable research area of QECCs. To construct QECCs, firstly the corresponding symplectic self-orthogonal codes should be constructed, and then determine the parameters of the constructed QECCs from the symplectic self-orthogonal code and its dual code.

\section{METHODS OF WEIGHT CALCULATION FOR SYMPLECTIC CODES}

As the symplectic weight is obviously different from that of classical Hamming weight, hence the present existed methods and softwares to calculate Hamming weight and determine Hamming-weight polynomial can't be directly applied to determine the symplectic-weight polynomial of symplectic codes. And furthermore, the determination of the symplectic weight and the symplectic-weight polynomial is more complex than that of the Hamming weight and the Hamming-weight polynomial. Hence a fast algorithm to determine the symplectic-weight polynomial of a symplectic code $C$ is an urgent task in symplectic code research.

Suppose the generator matrix of a symplectic code $C$ is

$$
G=\left(\begin{array}{c}
\alpha_{1} \\
\alpha_{2} \\
\vdots \\
\alpha_{m}
\end{array}\right)=\left(\begin{array}{c|c}
a_{1} & b_{1} \\
a_{2} & b_{2} \\
\vdots & \vdots \\
a_{m} & b_{m}
\end{array}\right)
$$


where $\alpha_{i} \in F_{2}^{2 n}, a_{i}=\left(a_{i 1}, a_{i 2}, \cdots, a_{i n}\right) \in F_{2}^{n}, b_{i}=$ $\left(b_{i 1}, b_{i 2}, \cdots, b_{i n}\right) \in F_{2}^{n} \quad, \quad i=1,2, \cdots, m$. Then each codeword in the symplectic code can be obtained by a binary linear combination of the rows in $G$.

(1)Statement of the problem

Let the symplectic code $C=[2 n, m]_{s}$, when the dimension $m$ is relatively small, all the codewords of $C$ can be obtained by a linear combination of the rows of its generator matrix $G$. But when $m$ is larger, this method is ineffective, and can't be applied to research on quantum coding theory.

As the sum of the dimension of the symplectic code $C$ with its symplectic dual code $C^{\perp s}$ equals to $2 n$, we try to convert the determination of the symplectic-weight polynomial of a high-dimension symplectic code to that of acorresponding low-dimension symplectic code. In the following, we always assume $C$ be a low-dimension symplectic code, and $C^{\perp s}$ is the corresponding highdimension symplectic code.

Let the symplectic-weight polynomial of symplectic code $C=[2 n, m]_{s}$ be $W_{s}(z)=\sum_{i=0}^{n} A_{i} z^{i}$, the symplecticweight polynomial of its dual code $C^{\perp s}$ be $B_{s}(z)=\sum_{i=0}^{n} B_{i} z^{i}$. From Ref.[3], we have :

$$
\begin{aligned}
& B(z)=\frac{1}{|C|}(1+3 z)^{n} A\left(\frac{1-z}{1+3 z}\right) \\
& =\frac{1}{|C|}\left(A_{0}(1+3 z)^{n}+A_{1}(1+3 z)^{n-1}(1-z)+\cdots\right. \\
& \quad+A_{i}(1+3 z)^{n-i}(1-z)^{i}+\cdots+A_{n}(1-z)^{n} .
\end{aligned}
$$

(2) Solution of the Problem :

For $i=0,1,2, \cdots, n$, let

Then

$$
(1+3 z)^{n-i}(1-z)^{i}=1+c_{i 1} z+c_{i 2} z^{2}+c_{i 3} z^{3}+\cdots
$$

$$
\begin{aligned}
& c_{i 1}=\left(\begin{array}{c}
n-i \\
1
\end{array}\right) 3-\left(\begin{array}{l}
i \\
1
\end{array}\right) \\
& c_{i 2}=\left(\begin{array}{c}
n-i \\
2
\end{array}\right) 3^{2}-\left(\begin{array}{c}
n-i \\
1
\end{array}\right) 3\left(\begin{array}{l}
i \\
1
\end{array}\right)+\left(\begin{array}{l}
i \\
2
\end{array}\right) \\
& c_{i 3}=\left(\begin{array}{c}
n-i \\
3
\end{array}\right) 3^{3}-\left(\begin{array}{c}
n-i \\
2
\end{array}\right) 3^{2}\left(\begin{array}{l}
i \\
1
\end{array}\right)+\left(\begin{array}{c}
n-i \\
1
\end{array}\right) 3\left(\begin{array}{l}
i \\
1
\end{array}\right)-\left(\begin{array}{l}
i \\
3
\end{array}\right)
\end{aligned}
$$

Following we explicitly illustrate the determination of the coefficent $c_{i 3}$ of $z^{3}$ as an example to show how to determine all the coefficients $c_{i k}$.
If $k>m$, let $\left(\begin{array}{c}m \\ k\end{array}\right)=0$. Then $c_{i 3}$ can be written in the form:

$$
\begin{aligned}
c_{i 3} & =\left(\begin{array}{c}
n-i \\
3
\end{array}\right) 3^{3}\left(\begin{array}{l}
i \\
0
\end{array}\right)(-1)^{0}+\left(\begin{array}{c}
n-i \\
2
\end{array}\right) 3^{2}\left(\begin{array}{l}
i \\
1
\end{array}\right)(-1)^{1} \\
& +\left(\begin{array}{c}
n-i \\
1
\end{array}\right) 3^{1}\left(\begin{array}{l}
i \\
2
\end{array}\right)(-1)^{2}+\left(\begin{array}{c}
n-i \\
0
\end{array}\right) 3^{0}\left(\begin{array}{l}
i \\
3
\end{array}\right)(-1)^{3}
\end{aligned}
$$

and the coefficient of $z^{k}$ can be written in the form

$$
\begin{aligned}
& c_{i k}=\left(\begin{array}{c}
n-i \\
k
\end{array}\right) 3^{k}\left(\begin{array}{l}
i \\
0
\end{array}\right)(-1)^{0}+\left(\begin{array}{c}
n-i \\
k-1
\end{array}\right) 3^{k-1}\left(\begin{array}{l}
i \\
1
\end{array}\right)(-1)^{1} \\
& +\left(\begin{array}{c}
n-i \\
k-1
\end{array}\right) 3^{k-2}\left(\begin{array}{l}
i \\
2
\end{array}\right)(-1)^{2}+\cdots+\left(\begin{array}{c}
n-i \\
0
\end{array}\right) 3^{0}\left(\begin{array}{l}
i \\
k
\end{array}\right)(-1)^{k}
\end{aligned}
$$

Using all the $c_{i k}(i=1,2, \cdots n, k=1,2, \cdots, n)$, one can composed a matrix of size $(n+1) \times(n+1)$

$$
M_{C}=\left(\begin{array}{llll}
c_{01} & c_{02} & \cdots & c_{0 n} \\
c_{11} & c_{12} & \cdots & c_{1 n} \\
\cdots & \cdots & \cdots & \cdots \\
c_{n 1} & c_{n 2} & \cdots & c_{n n}
\end{array}\right)
$$

Multiply the $i$ row of $M_{c}$ by $A_{i}$, sum up all the elements in the newly obtained matrix we have the coefficients $B_{0}, B_{1}, \cdots, B_{n}$ of $\quad+\mathbb{A}(3) A_{1} 1(+$ $3 z)^{n-1}(1-z)+\cdots+A_{n}(1-z)^{n}$, i.e.

$$
2^{m}\left(B_{0}, B_{1}, \cdots, B_{n}\right)=\left(A_{0}, A_{1}, \cdots, A_{n}\right) M_{C}
$$

The matrix $M_{C}$ is independence from symplectic code $C$, and determined by $n$ uniquely. From the symplecticweight polynomial of arbitrary given length $n$ and symplectic code $C=[2 n, m]_{s}$, we can quickly obtain the symplectic-weight polynomial of the dual code $C^{\perp s}=[2 n, 2 n-m]_{s}$. The above given method is a faster algorithm to determine the symplectic-weight polynomial of the symplectic dual code than by its usual definition.

IV. IDENTIFICATION OF SELF-ORTHOGONAL CODE AND DETERMINATION OF GENERATOR MATRIX OF ITS DUAL CODE

If $G$ is a generator matrix of a classical code $C$, then $C$ is self-orthogonal if and only if $G G^{T}=0$. If $G=\left(I_{m}, A\right)$ where $I_{m}$ be identity matrix, then $C^{\perp s}$ has generator matrix $G=\left(-A^{\prime}, I_{n-m}\right)$. All these are well known facts [3]. For symplectic code, analogous results can also be derived as follows. 


\section{A. Dentification of symplectic self-orthogonal code}

Let the generator matrix of the symplectic code $C=[2 n, m]_{s} \quad$ be $G$ and the $i$ row of $G$ be $\alpha_{i}=\left(a_{i} \mid b_{i}\right)$. Define $\hat{\alpha}_{i}=\left(b_{i} \mid a_{i}\right)$ for $1 \leq i \leq m$ and $\hat{G}$ be the the matrix whose $i$ row is $\hat{\alpha}_{i}$. The code $C$ is symplectic self-orthogonal if and only if arbitrary two rows of $G$ is symplectic orthogonal, i.e. the following hold

$$
\begin{aligned}
& \left(\alpha_{i}, \alpha_{j}\right)=0 \Leftrightarrow a_{i} b_{j}^{T}+b_{i} a_{j}^{T}=0 \\
& \Leftrightarrow a_{i} b_{j}^{T}+b_{i} a_{j}^{T}=\left(a_{i}, b_{i}\right)\left(\begin{array}{l}
b_{j}^{T} \\
a_{j}^{T}
\end{array}\right) \\
& \Leftrightarrow \alpha_{i} \hat{\alpha}_{j}^{T}=0
\end{aligned}
$$

where $1 \leq i, j \leq m$.

The above identities can be stated as: $C$ is a symplectic self-orthogonal code if and only if $G \hat{G}^{T}=0$, where $\hat{G}$ is the matrix obtained by exchanging the left and right part of the row vectors in $G$.

Next, suppose $G$ generates a symplectic self-orthogonal code $C$, the generator matrix of $C^{\perp s}$ is $B$. Then how to determine $B$ ?

\section{B. Determination of the generator matrix of symplectic dual codes}

Suppose after row transformation (and also column transformation when necessary), we can transform $G$ into $\left(I_{m} A_{1} \mid A_{2}\right)=\left(I_{m} A\right)$, where $A_{1}$ has $n-m$ columns, and $A_{2}$ has $n$ columns, $A=\left(A_{1} \mid A_{2}\right)$. This process can be describe as $P G Q=\left(I_{m} A_{1} \mid A_{2}\right)$, where $P$ is an invertible matrix and $Q$ is a permutation matrix.

Now suppose $G=\left(I_{m} A_{1} \mid A_{2}\right)$, from $G \hat{B^{T}}=0$, we have

$$
\begin{aligned}
& \hat{B}=\left(\begin{array}{ccc}
A_{1}^{T} & I_{n-m} & O_{n-m, n} \\
A_{2}^{T} & O_{n,(n-m)} & I_{n}
\end{array}\right) \\
& B=\left(\begin{array}{ccc}
O_{(n-m), n} & A_{1}^{T} & I_{n-m} \\
I_{n} & A_{2}^{T} & O_{n,(n-m)}
\end{array}\right)
\end{aligned}
$$

It is trivial to check $\hat{G B^{T}}=0$.
C. Algorithm to obtain $\left(I_{m} A_{1} \mid A_{2}\right)=\left(I_{m} A\right)$ from

$G_{m, 2 n}$

step1: Search for a nonzero element $a_{i, k}=1$, where $i \leq k \leq m$, then $\alpha_{i} \leftrightarrow \alpha_{k}$, go to step2; If $a_{i, k}=0$, $i \leq k \leq m$, then search for a nonzero element $b_{k, n}=1$ where $i \leq k \leq m$, then $\alpha_{i} \leftrightarrow \alpha_{k}$ and exchange $i+n$ and $i$ column of $G$, go to step2.

Step2: Eliminate all the nonzero elements of the $i+1$ to $m$ row in $i$ column by $a_{i i}$.

Step2: The element $a_{i, k}$ can be changed to zeros through element row transformation for $i+1 \leq k \leq m$.

Step3: Eliminate all the nonzero elements of the $i-1$ to 1 row in $i$ column by $a_{i i}$.

Step3: The element $a_{i, k}$ can be changed to zeros through element row transformation for $k$ from $i+1$ to 1 .

Thus we can prove that $G \hat{B^{T}}=0$.

\section{IDENTIFICATION OF IMPURE CODES AND EXAMPLES}

In the past few decades, almost all the quantum codes constructed are pure codes except for few sporadic cases. What are the characteristics of impure codes ? What impure codes have performance better than pure codes? These problems are still remained unsolved yet.

When using a symplectic self-orthogonal code $C$ to construct qutum code $Q$, the minimum distance $d$ of the quantum code is the minimum distance in $C^{\perp s} \backslash C$. If $d<d(C)$, then the code $Q$ and $C$ are called puer codes; otherwise impure. code

Purity Identification of symplectic self-orthogonal

Let $C$ be a symplectic self-orthogonal code, the symplectic weight polynomial of $C$ is $1+a_{d} z^{d}+\cdots$ $+a_{d+i} z^{(d+i)}+\cdots a_{n} z^{n}$, the symplectic-weight polynomial of $C^{\perp s}$ is $1+b_{d} z^{d^{\prime}}+\cdots b_{n} z^{n}$, where $a_{d} \neq 0$ and $b_{d^{\prime}} \neq 0$. If $d<d^{\prime}$, then $C$ is pure. If $d=d^{\prime}$, then $C$ is impure.

Based on the theory foundation and search algorithm in previous sections, we obtain classes of symplectic selforthogonal codes, but most of them are pure codes, only a few are impure symplectic self-orthogonal codes. These 
results show that when $m<2$, impure symplectic selforthogonal codes don't exist; when $3 \leq m \leq 7$ impure symplectic self-orthogonal codes may exist [4-5]. When $m=5, n=6$ there are two impure symplectic selforthogonal codes, this is consistent with [2]. Following we give three new results correspond to optimal impure quantum codes.

Let

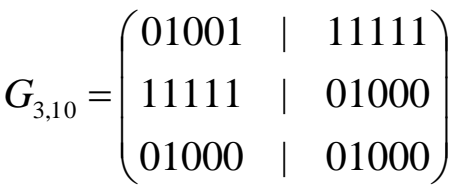

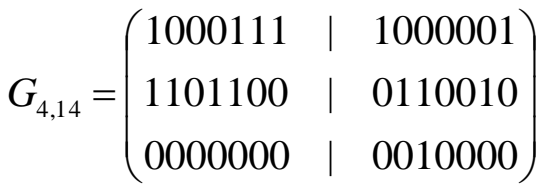

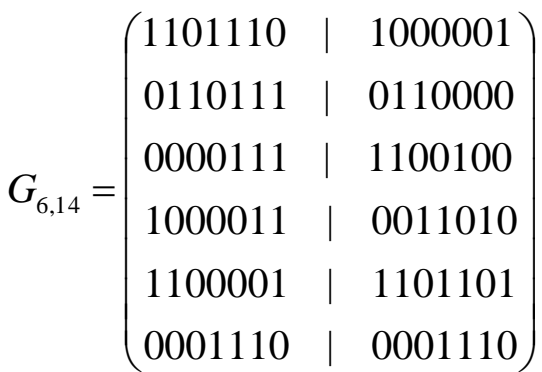

We will show each these three matrices generate an impure symplectic self-orthogonal code.

Example 1. $[10,3]_{s}$ impure symplectic self-orthogonal code.

Let $C=[10,3]_{s}$ be the code generated by $G=G_{3,10}$.It is easy to check $G \hat{G^{T}}=0$, hence $C$ is a symplectic selforthogonal code. $C$ has symplectic-weight polynomial $1+z+3 z^{4}+3 z^{5}$. The symplectic-weight polynomial of its symplectic-dual code $C^{\perp s}$ is $1+z+18 z^{2}+42 z^{3}+\cdots$. Thus, $C$ is an impure code, and the impure quantum code [[5,2,2]] stabilized by $C$ is optimal.

Example 2. $[14,4]_{s}$ impure symplectic self-orthogonal code.

Let $C=[14,4]_{s}$ be the code generated by $G=G_{4,14}$.
It is easy to check $G \hat{G}^{T}=0$, hence $C$ is a symplectic self-orthogonal code. $C$ has symplectic-weight polynomial $1+z+z^{4}+5 z^{5}+6 z^{6}+2 z^{7}$. The symplectic-weight polynomial of its symplectic-dual code $C^{\perp s}$ is $1+z+17 z^{2}+89 z^{3}+\cdots$. Thus, $C$ is an impure code, and the impure quantum code $[[7,3,2]]$ stabilized by $C$ is optimal.

Example 3. $[14,6]_{s}$ impure symplectic self-orthogonal code.

Let $C=[14,6]_{s}$ be the code generated by $G=G_{6,14}$.

It is easy to check $G \hat{G}^{T}=0$, hence $C$ is a symplectic self-orthogonal code. $C$ has symplectic-weight polynomial $1+z^{2}+2 z^{2}+7 z^{4}+24 z^{5}+23 z^{6}+6 z^{7}$. The symplectic-weight polynomial of its symplectic-dual code $C^{\perp s}$ is $1+z^{2}+20 z^{3}+43 z^{4}+\cdots$. Thus, $C$ is an impure code, and the impure quantum code $[[7,1,3]]$ stabilized by $C$ is optimal.

\section{ACKNOWLEDGMENT}

The authors would like to thank Prof. Ruihu Li for his guidance, and Doctor Xu Gen for useful conversation. This work was support by Student Innovation Project of China.

\section{REFERENCES}

[1] D. Gottesman, Stabilizer codes and quantum error correction, Ph.D thesis, California Institute of Technology, 1997.

[2] A. R. Calderbank, E. M. Rains, P. W. Shor and N. J. A. Sloane, Quantum error-correction via codes over GF(4), IEEE. Trans. Inform. Theory, vol.56, pp. 1369-1387, 1998.

[3] F. Feng and H.Chen, Quantum Error-correcting Codes (in Chinese), Science Press, Beijing, 2010.

[4] Z. Li and L. Xing, On a problem concerning the quantum Hamming bound for impure quantum codes, IEEE. Trans. Inf. Theory, vol.56, pp. 4731-4734, 2010.

[5] P. Sarvepalli and A. Klappenecker, Degenerate quantum codes and the quantum Hamming bound, Phys. Rev. A, vol. 81, 032318, 2010.

[6] J. Bierbrauer, Y. Edel, G. Faina, S. Marcugini and F. Pambianco, Short additive quaternary codes, IEEE. Trans. Inform. Theory, vol. 55, 952-954, 2009. 